ARTICLE

\title{
Large scale genome reconstructions illuminate Wolbachia evolution
}

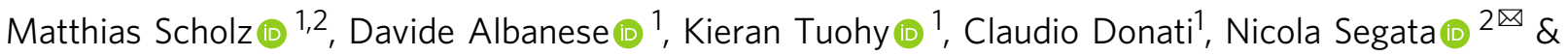 \\ Omar Rota-Stabelli (iD) 1,3凶
}

Wolbachia is an iconic example of a successful intracellular bacterium. Despite its importance as a manipulator of invertebrate biology, its evolutionary dynamics have been poorly studied from a genomic viewpoint. To expand the number of Wolbachia genomes, we screen over 30,000 publicly available shotgun DNA sequencing samples from 500 hosts. By assembling over 1000 Wolbachia genomes, we provide a substantial increase in host representation. Our phylogenies based on both core-genome and gene content provide a robust reference for future studies, support new strains in model organisms, and reveal recent horizontal transfers amongst distantly related hosts. We find various instances of gene function gains and losses in different super-groups and in cytoplasmic incompatibility inducing strains. Our Wolbachiahost co-phylogenies indicate that horizontal transmission is widespread at the host intraspecific level and that there is no support for a general Wolbachia-mitochondrial synchronous divergence.

\footnotetext{
${ }^{1}$ Research and Innovation Centre, Fondazione Edmund Mach (FEM), San Michele all'Adige, Italy. ${ }^{2}$ Department CIBIO, University of Trento, Trento, Italy.

${ }^{3}$ Present address: Centre Agriculture Food Environment (C3A), University of Trento, Trento, Italy. ${ }^{凶}$ email: nicola.segata@unimi.it; omar.rota@fmach.it
} 
$\mathrm{N}$ ature is filled with exemplar cases of symbiotic interaction between bacteria and multicellular eukaryotes. While bacteria in such partnerships benefit from a protected environment, hosts are endowed with different fitness advantages such as key nutrients and antimicrobial protection ${ }^{1-3}$. The most studied and evolutionary remarkable case of symbiotic interaction is that of Wolbachia, an alpha-proteobacterium which infects animal cells ${ }^{4}$ and specifically invertebrates belonging to the Ecdysozoa, the superphylum encompassing nematodes, insects, and other arthropods. Wolbachia infection is extremely widespread, and estimated to occur in up to $40-50 \%$ of terrestrial arthropods ${ }^{6,7}$, implying more than a million infected host species worldwide.

One peculiarity of Wolbachia is that it not only provides fitness advantages to the host by conferring for example microbial protection, but it can also manipulate the host reproductive biology to increase its own chances of transmission using for example sperm-egg incompatibility of hosts upon asymmetric Wolbachia infections, known as cytoplasmic incompatibility, $\mathrm{CI}^{4}$. These characteristics have heightened interest in using Wolbachia to control mosquitoes arboviruses ${ }^{8,9}$ and reduce pest populations via $\mathrm{CI}^{10}$. These manipulating experiments require a fine understanding of Wolbachia biology and diversity, and genomics can be a first step in this direction. Indeed, the long sought effectors of CI have been identified only recently after decades of research using comparative genomics ${ }^{1,12}$, but the major current limitation in Wolbachia genomics is that sequenced genomes are available from only a few hosts and strains (43 reference genomes at January 2018, Supplementary Data 3).

Although the main route of a Wolbachia transmission is from mother to offspring, horizontal transfer between host species (interspecific transmission) and loss of infection is common to the point that Wolbachia and host phylogenies do not agree ${ }^{13,14}$. The degree of Wolbachia intraspecific horizontal transfer (transmission between individuals of the same host species) is less documented but has been shown to play a key epidemiological role for example in cherry fruit flies ${ }^{15}$. Co-phylogenetic studies based on genomic data have found no clear evidence of intraspecific transmission in the fly $D$. melanogaster ${ }^{16}$ nor in the nematode $O$. volvolus ${ }^{17}$ and could not address this issue in $D$. simulans due to lack of phylogenetic signal ${ }^{18}$. Understanding whether Wolbachia generally transmits only vertically intraspecifically would require whole-genome analyses of more host populations as PCR based screening such as Multi Locus Sequence Typing (MLST) does not have sufficient discriminating power $^{19,20}$.

Wolbachia, like most other endosymbionts, cannot be cultured in isolation. For this reason, and even when protocols for Wolbachia enrichment are used ${ }^{21}$, the host genome is routinely cosequenced in the same experiment. This creates potential coverage and contamination issues as the host genome is typically two to three orders of magnitude larger than that of the symbiont. This however also presents an opportunity as shotgun sequences targeting animals may serendipitously contain traces of their symbionts which might then be extracted to assemble genomes. Recovery of Wolbachia genomic DNA present in Drosophila sequencing data $16,18,22$ has previously been demonstrated, including a recent effort to screen available sequencing projects using three Wolbachia genes as queries ${ }^{23}$.

To increase the number of available Wolbachia genomes, here we present a comprehensive and systematic screening of deposited Ecdysozoa sequencing projects. We use a novel combination of assembly-free ${ }^{24}$ and assembly-based tools (see Methods) to recover whole-genome information for more than 1000 newly metagenome-assembled genomes (MAGs). Although MAGs cannot reach the quality of isolate genome sequencing which is unavailable for intracellular parasites, this large catalog of Wolbachia MAGs allows us to infer robust phylogenies, identify new variants, build host population-level datasets, and ultimately clarify some open questions concerning Wolbachia evolution.

\section{Results}

Expanding Wolbachia genomics with 1,005 newly assembled genomes. We screened for the presence of Wolbachia sequences a

Host phylogeny

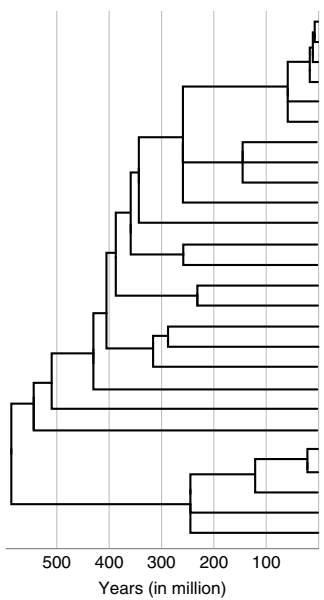

Prevalence

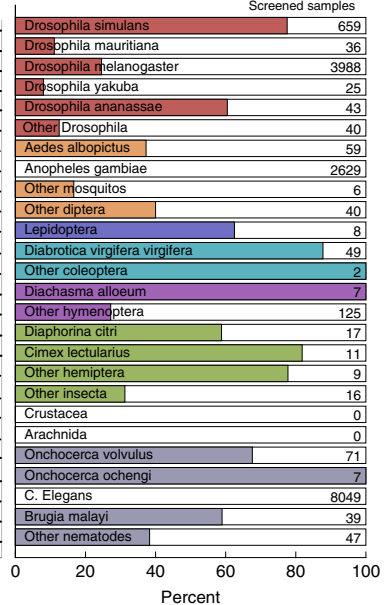

Estimated titer

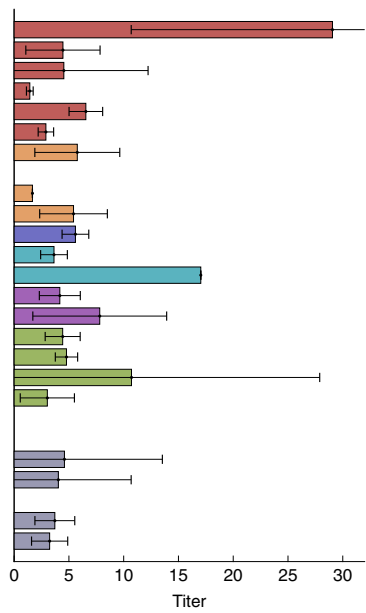

b

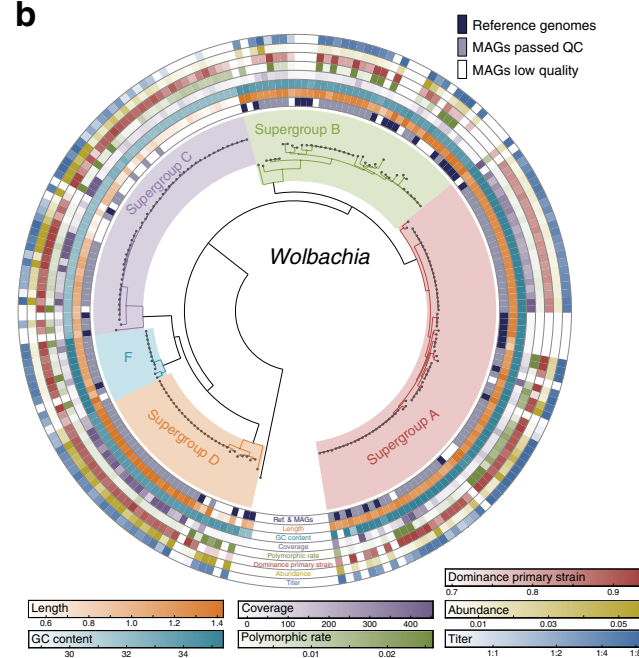

Fig. 1 Prevalence, titer, and genome characteristics of Wolbachia in the analyzed samples. a Host related prevalence and estimated titer of more than 30,000 screened samples in relation to the host species phylogeny based on Rota-Stabelli et al. ${ }^{5}$. Wolbachia prevalence is based on the fraction of host samples in which Wolbachia strains could be detected at 1X or higher coverage using PanPhIAn. Statistics are reported only for host species with at least 7 samples. Infectious titers of Wolbachia cells per host cell are estimated from the fraction of reads and normalized by Wolbachia and host genome size taken from http://www.genomesize.com/. Error bars indicate the standard deviation over all titers of samples from the same host species. b PhyloPhIAn tree based on 1166 assembled genomes; reported for each genome are genome length, nucleotide composition in terms of $\mathrm{G}+\mathrm{C}$ content, depth of coverage, fraction of nucleotide polymorphisms and dominance of the primary strain, relative read abundance, and estimated titer (normalized abundance). The large branches of $D$. melanogaster and $D$. simulans are excluded and duplicated sequences are not shown for clarity. 
in more than 70TB of raw DNA sequencing data from 31,825 publicly available genome sequencing samples from more than 500 putative host species ranging from nematodes and insects to crustaceans, but also including various non-ecdysozoan species (Supplementary Data 4). Raw reads were mapped against a set of 43 decontaminated available Wolbachia draft genomes using a $\mathrm{PanPhlAn}{ }^{24}$-based pipeline (see Methods).

We found a total of 1793 samples (5.63\%) positive for Wolbachia. This general low infection frequency is due to many samples being from model organisms including Aedes aegypti, Caenorhabditis elegans and Anopheles gambiae which are supposed to be Wolbachia-free or only very rarely infected with Wolbachia $^{25}$. We verified the exact identity of all host species by reconstructing their $18 \mathrm{~S}$ rRNA small subunit (see Supplementary Information and Supplementary Data 5). We found large differences in the level of infection frequency (prevalence) in the various hosts (Fig. 1a and Supplementary Data 4)) with values ranging from $100 \%$ in Diachasma alloeum to $<5 \%$ in Nasonia vitripennis. Large differences exist even within the same genus. Wolbachia prevalence in Drosophila simulans and D. ananassae is twice of that in D. melanogaster and almost ten times higher than in other Drosophila species, confirming observations of similar large variations reported in other genera ${ }^{26}$. Despite consistency in the analytical pipeline used here across samples, these quantitative prevalence results should be taken with care because of likely sampling biases not traceable in sequence archives such as the presence of antibiotic-treated host strains, differences in DNA extraction protocols, and natural versus laboratory environments. The number of Wolbachia cells per host cell (normalized by genome size to approximate the actual titer) was also quite dissimilar with most estimates ranging from 3:1 to 10:1 (Wolbachia cells: host cells); exceptions are D. simulans (infected by wRi, 25:1) and the coleopteran Callosobruchus chinensis (infected by our newly assembled wCch 17:1).

We then metagenomically assembled 1166 genomes-called metagenome-assembled genomes (MAGs)-from our 1793 positive samples (see "Methods"), by selecting those characterized by high Wolbachia coverage. We verified that our Wolbachia MAGs did not contain host genome integrated fragments (see Supplementary Information), and excluded contaminations from other bacteria. By mapping all MAGs against nearly 100,000 bacteria reference genomes, we found only one instance of contamination. Using a genome-wide maximum-likelihood approach with PhyloPhlAn ${ }^{27}$ (Fig. 1b) we revealed a highly heterogeneous distribution of various genetic characteristics (genome length, nucleotide composition) and biological traits (normalized abundance: see Methods and Supplementary Fig. 2). In some cases the phylogenetic structure of Wolbachia could be linked with certain traits as in the case of supergroup C characterized by low GC content and reduced genome length, or supergroup D characterized by high abundance.

We defined MAGs quality based on four main criteria (see "Methods" and Fig. 1b) as a strict control to retain a total of 1005 Wolbachia MAGs, which we have used to infer a whole-genome phylogeny based on the alignment of 316 core genes (Fig. 2a). Although MAGs are inherently less accurate than genomes obtained by isolate sequencing, the resulting maximum-likelihood tree is overall very robust with most nodes receiving full $(100 \%$ bootstrap) support. We complemented this phylogeny with an analysis of the presence and absence of the 6376 gene families composing the pangenome of the 1793 Wolbachia-positive samples using PanPhlAn ${ }^{24}$ (Fig. 2b). The two trees show a similar topology as further indicated by comparison of branch lengths (Fig. $2 \mathrm{c}, \rho=0.94, p<1 \mathrm{E}-50$, Spearman correlation). The position of supergroups $\mathrm{C}$ and $\mathrm{D}$ is however an exception. This is likely due to shared functional properties between F, A, and B, which may have promoted a Long Branch Attraction artifact ${ }^{28}$ of $\mathrm{C}$ and $\mathrm{D}$ with functionally distant outgroups. Overall, our analysis increased the diversity of available Wolbachia genomes by more than $60 \%$ reaching 1166 Wolbachia genome and increased by more than $50 \%$ the number of host species with an assembled Wolbachia genome (from 33 as of July 2018 to 55), providing an useful dataset for comparative genomic and phylogenetic studies (Supplementary Data 5).

The catalog of reconstructed strains expands the Wolbachia sampled diversity. Our Wolbachia genome resource contains new MAGs of previously described data in four host species (Drosophila melanogaster, D. simulans, D. ananassae, Onchocerca vulvus $)^{16-18,29}$ and crucially expands Wolbachia diversity with newly assembled genomes from both known and unknown hosts (white circles in Fig. 2a). For example, we enlarged the genomic diversity in D. melanogaster and D. simulans by adding new genomes to reach more than 500 quality controlled genomes each, including the Wolbachia genomes for D. mauritiana ${ }^{30}, D$. yakuba and $D$. santomea ${ }^{31}$. We similarly provide additional assembled genomes for various nematodes of medical relevance such as Wuchereria bancrofti and Brugia pahangi, and genomes in insect of economic importance such as the corn rootworm Diabrotica virgifera and the bedbug Cimex lecturalis. These collections of Wolbachia genomes from the same host (black circles in Fig. 2a) provided us with data for Wolbachia intra-host population studies, as addressed below. We also assembled many genomes for unrepresented insect orders Lepidoptera (e.g: butterflies Polygonia c-album and Pararge aegeria), Hemiptera (e.g., the pest species Homalodisca vitripennis and Dactylopius coccus), and Hymenoptera (e.g: parasitoid wasps Diachasma alloeum and two bee species). We assembled the first Wolbachia genome from an arachnid host, the mite Tetranychus urticae. Overall, the majority of our newly assembled genomes are from insects and nematodes because hosts such as terrestrial crustaceans and arachnids are poorly represented in sequence archives. A detailed description of newly detected strains is given in Supplementary information.

Evolutionary scenarios highlighted by the new Wolbachia MAGs. We found evidence of high host intraspecific Wolbachia genetic diversity. In some cases, this variability was geographically linked as for the gall wasp Biorhiza pallida and the ant Cardiocondyla obscurior (in purple in supergroup A, Fig. 2b), or in the medical relevant tiger mosquito Aedes albopictus and tsetse fly Glossina morsitans (orange in, respectively, supergroup A and B, Fig. 2b). We also found new strains in D. simulans which are robustly separated (high bootstrap support) from known variants resident in this host such as the $w$ Au-like strains in the box of Fig. 2a. This finding points toward the peculiar role of D. simulans as a reservoir of Wolbachia diversity in nature with at least seven distinct Wolbachia genome types observed in this species.

In some cases we identified Wolbachia strains very closely related to well-known reference Wolbachia genomes that are however present in unexpected hosts. We found, for example, a genome from the robber fly Holcocephala fusca having a 99.52\% core genetic identity with $w \mathrm{Mel}$ of $D$. melanogaster: Although $18 \mathrm{~S}$ rRNA gene screening confirms this SRA as Holcocephala, we found some reads with high similarity to the cytochrome oxidase subunit I gene (COI) of hoverflies. We therefore cannot exclude that our reconstructed Wolbachia genome is not from the robber fly itself, but from a hoverfly prey. Because we can exclude contamination from a Drosophila prey (Supplementary Information), this strain likely indicates a recent horizontal transfer involving distant hosts and showcases the complexity of the 


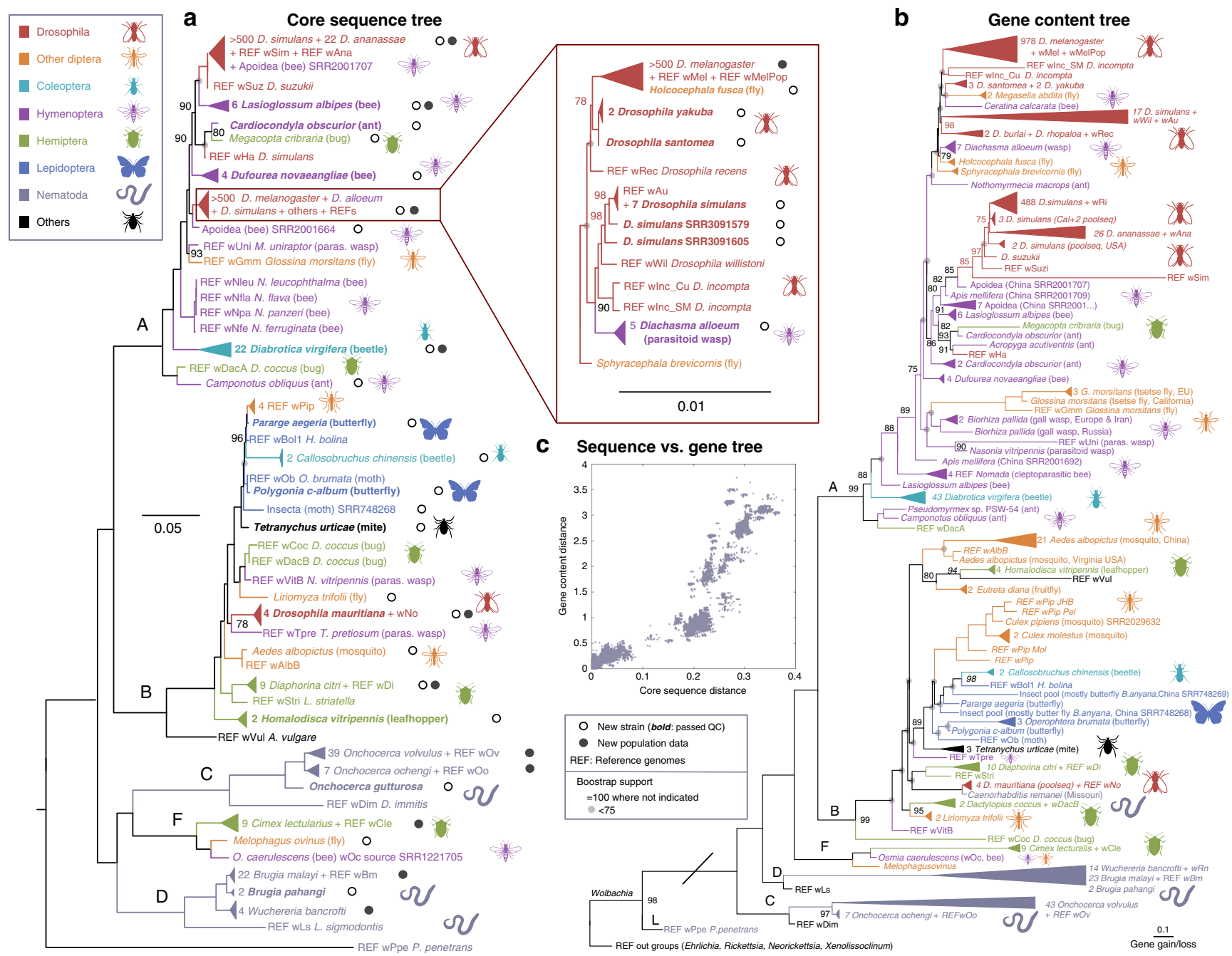

Fig. 2 Whole-genome phylogenies of Wolbachia. a Maximum likelihood tree of 313 core genes alignment employing 339,972 nucleotide positions from 1161 newly assembled genomes (1005 genomes complied with our quality criteria plus 156 of core gene quality, see "Methods" section and Supplementary Data 2). Larger clades have been collapsed and their subtrees are represented by triangles. Bold host species indicate assembled genomes that fulfilled our quality requirements. The complete tree including support values is available in Newick format (Supplementary Data 1). b Functional potential tree based on presence and absence of 6376 gene families in 1793 Wolbachia positive samples as inferred by PanPhIAn. Gene families present in $<5$ Wolbachia positive samples were not considered. Both trees were inferred with RAxML using the GTR+G model of replacement and the BINGAMMA model, respectively, in both cases bootstrapping with 100 pseudo-replicates (for support see legend). c Scatterplot showing pairwise distances between all possible genome pairs in the core gene sequences (tree in a) versus identical pairs in the binary tree based on gene presence (b). Animal silhouettes have been created by MS.

Wolbachia-Drosophila symbiotic models. We further identified a dubious new strain in the nematode Caenorhabditis remanei with a $98.04 \%$ identity in gene content with $w$ No of $D$. mauritiana (Fig. 2b). While we could verify the host based on $100 \%$ similarity with the annotated COI gene of $C$. remanei, we also found some reads covering a portion of $D$. mauritiana COI. Since no Wolbachia has ever been found in the Caenorhabditis genus, it is possible that this Wolbachia is the result of some kind of contamination. Indeed, the genome from this sample did not pass our quality control and was considered only for our gene-content tree (Fig. $2 \mathrm{~b}$ and not Fig. 2a). These two cases highlight the difficulty in determining the exact source of some samples when reconstructing endosymbiont genomes using a metagenomic approach.

As expected from a symbiont that can transfer horizontally between host species, we did not observe a strict or meaningful phylogenetic clustering of hosts in our Wolbachia phylogenies. We however identify some peculiar patterns, for example a clustering of Lepidoptera and a concentration of Hymenoptera in group A (respectively blue and purple in Fig. 2). The latter have a clear paraphyletic distribution on the tree consistent with a scenario where Hymenoptera in general (and not only parasitoids $^{32}$ ) may have played a key role in the differentiation of Wolbachia supergroup A.

Lineage-specific acquisition of gene families in supergroups. Our functional pangenome analysis (Fig. 3a) showcased large gene content divergence even within supergroups (in particular A). We performed a more stringent host-specific functional enrichment analysis by selecting 989 genomes from 14 different hosts with multiple assemblies. Investigating the functional enrichments and depletions (enzyme groups) on the phylogeny (Fig. 3b and Supplementary Fig. 1) we found that some functionalities have been independently lost in different host-specific lineages including for example the UDP- $N$-acetylmuramate-alanine ligase and the Glycerol-3-phosphate acyltransferase. Conversely, the Holo-acylcarrier-protein synthase (EC 2.7.8.7) was instead acquired in the 


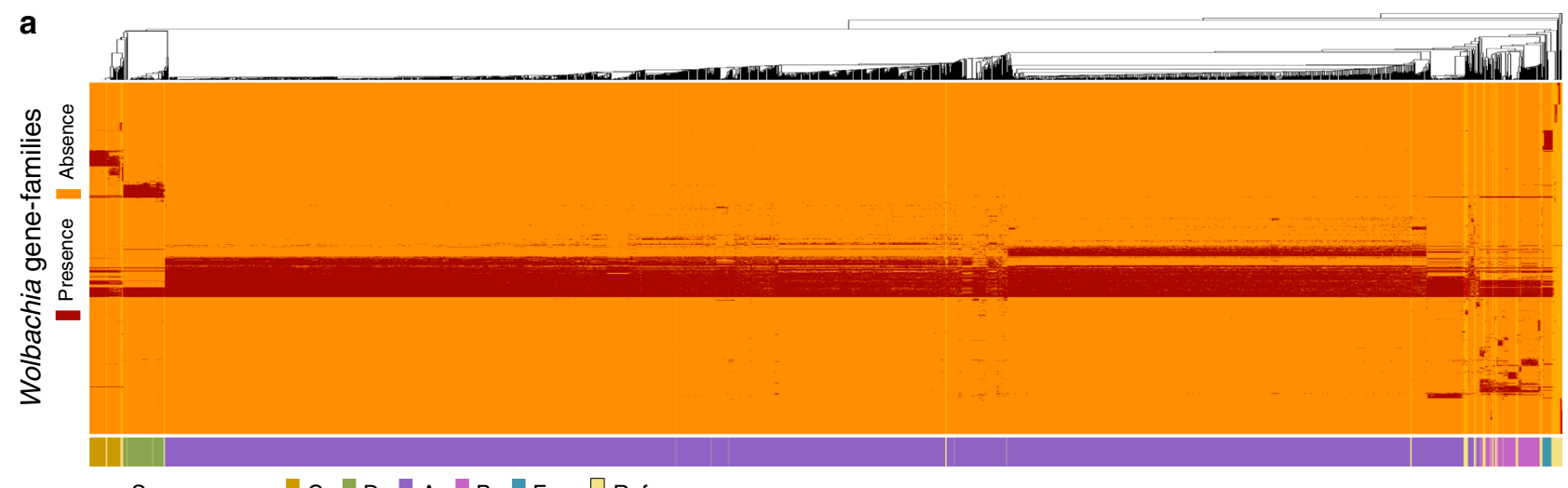

Supergroups $\mid \mathrm{C}\|\mathrm{D}\| \mathrm{A}\|\mathrm{B}\| \mathrm{F} \quad \square$ Ref. genomes

b Enzyme-catalyzed reactions

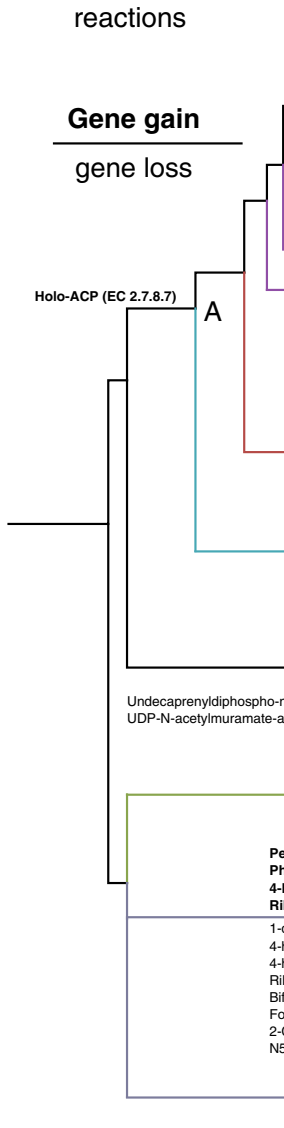

Drosophila melanogaster

Drosophila melanogas

Putative endonuclease (EC 3.1.4.4)
Drosophila simulans WAu-like

Diachasma alloeum

Isoleucine-trNA ligase (EC 6.1.1.5)

- Dufourea novaeangliae

Lasioglossum albipes

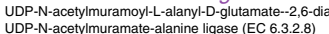

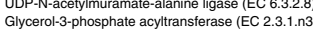

Drosophila simulans wRi-like

Ferredoxin-NADP reductase (FNR) (Fd-NADP(+) reductase) (EC 1.18.1.2)

NADH dehydrogenase subunit H (EC 1.6.99.5)

Drosophila ananassae
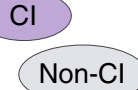

Non-Cl
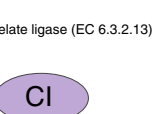

Diabrotica virgifera virgifera Diacyllycererl kinase (EC 2.7.1.1.107)
N-acetylyuramoyy-L-alanine amidase (EC 3.5.1.2.

B

Drosophila mauritiana

C

Cytoplasmic incompatibility $(\mathrm{Cl})$

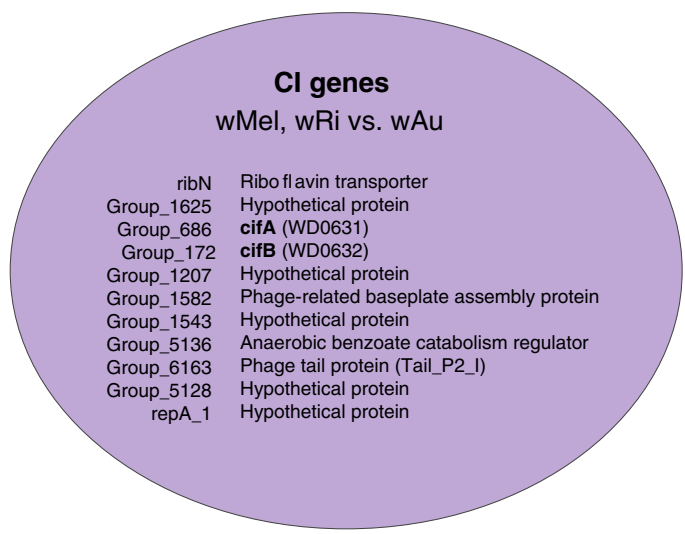

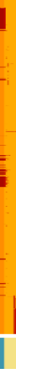


a
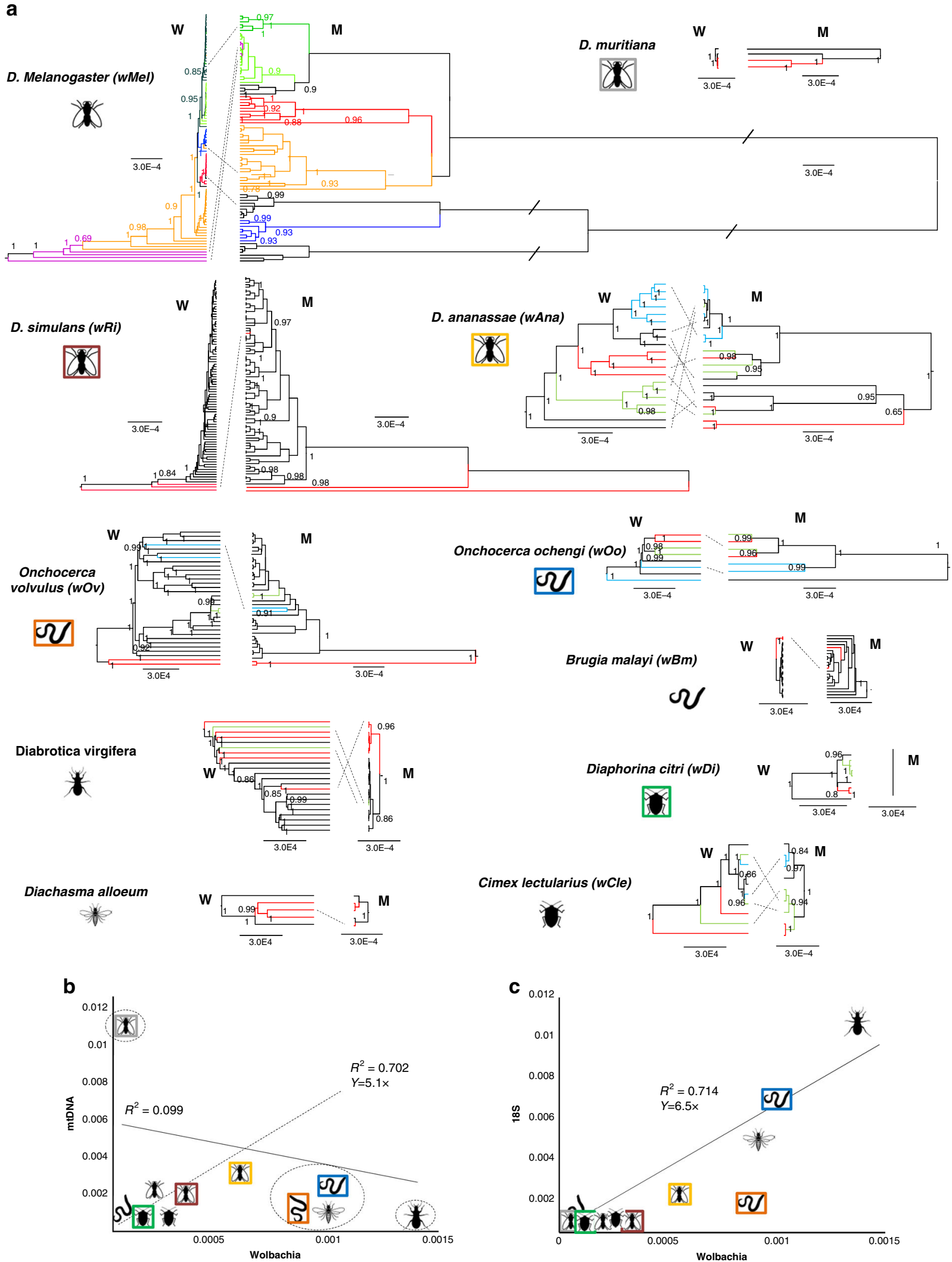

large panel of Wolbachia, our assumption was necessary to perform a pangenome analysis which is statistically robust against random sampling. We found that among the 11 candidate genes significantly enriched in CI inducing genomes (Fisher's exact test, $p<10^{-05}$, Bonferroni corrected, Supplementary Data 6), five of

them, including cif $A$ and $c i f B$ were previously identified by LePage et al. ${ }^{11}$. Our pangenome analysis reveals six additional genes with functional annotations related to, among others, riboflavin, benzoate, and a bacteriophage. While significantly enriched phage genes might not be surprising, as the CI loci are 
Fig. 4 Co-phylogenies of Wolbachia, mitochondria, and 185 in eleven host populations. a Bayesian consensus trees of Wolbachia (W) and corresponding mitochondrial host (M) genomes from eleven species. All trees have been rescaled to the same rate (0.0003 mut/site) and colors were added to aid comparisons. A bar on branches indicates that the branch has been halved in length in order to fit the figure. Nodes below posterior probability 0.85 are considered poorly supported, and they are not considered to report a phylogenetic incongruence (dotted lines). b Average genetic distances (mutations per site) of each of the Wolbachia populations against the average genetic distance of the corresponding host's mitochondrial genome. The dotted line is the regression in the absence of circled populations. c Average genetic distances of Wolbachia populations against the average genetic distance of the corresponding host's ribosomal 18S. Full detailed versions of the trees, together with all NCBI-SRA names and BEAST xml files and genetic distances can be found in Supplementary Information and Supplementary Data 1.

located in a prophage region, other candidates may play a role in CI biology and should be investigated further experimentally.

\section{Correspondence of co-phylogenies and intraspecific horizontal} transfers. Our extended genomes catalog allowed us to explore Wolbachia evolutionary dynamics within host populations, in particular, to assess the degree of intraspecific horizontal transmission. We used a reference-based mapping as in previous population studies ${ }^{16,18,32}$ to obtain longer Wolbachia genome alignments and assembled 1,149 mitochondrial genomes of the corresponding hosts to evaluate their co-phylogeny in eleven species (Fig. 4a).

Our co-phylogenies indicate a good correspondence between intraspecific host relationships and their corresponding Wolbachia: this is consistent with a prevalently vertical rather than horizontal inheritance as previously shown in D. melanogaster ${ }^{16}$. We found however numerous exceptions. All hosts species, except for the poorly sampled $D$. mauritiana, are characterized by one or more cases of phylogenetic incongruence which corresponds to likely cases of intraspecific horizontal Wolbachia transmission (dotted lines in Fig. 4a; the actual cases of horizontal transfer may be more than those apparent from our analyses because we considered only highly supported incongruences). For $D$. melanogaster and D. ananassae we observed some instances of horizontal transmission, in conflict with previous studies using less data ${ }^{16,29,32}$. We further found hints of peculiar inheritance mechanisms. Exceptional is the case of the bug D. citri which is characterized by clonal (identical) mitochondria, but whose Wolbachia population is well structured. This may reflect recent host infections from a pool of genetically diverse Wolbachia or a case of recent intraspecific transmission ${ }^{15}$. Of note is also the case of D. simulans whose Wolbachia are almost indistinguishable, while the host has some mitochondrial structure compatible with the recent rapid spread of $w \mathrm{Ri}$ within this host ${ }^{33}$. To check for possible contaminants that may have misled our co-phylogenies, we further explored possible host-derived sequences using the procedure of Chung et al. ${ }^{34}$ (see Methods) and found on average about $0.1 \%$ positions of potential sequence variant contamination across all assemblies. We detected various instances of increased sequence variations at $1-6 \%$ length, but only eight assemblies with increased coverage regions at $1-3 \%$ length mostly in nematode hosts. (Supplementary Data 5). Because Wolbachiahost lateral gene transfers (LGTs) shall generate a significant increase in coverage variation, we conclude that only eight of our MAGs are potentially affected by misleading signal related to LGT, None of these MAGs are involved in any of the putative within host transfers (dotted line of Fig. 4a) or exhibit any peculiar rate of evolution in Fig. 2a. However, because we found a certain level of genetic heterogeneity, we cannot completely exclude confounding factors from multiple Wolbachia infections in some of the MAGs. For D. ananassae we further manually excluded bias from $w$ AnaINTs (Wolbachia genomes integrated in host genomes) data accidentally included in our assemblies, but could not find fragments attributable to integrated Wolbachia (see Supplementary Information); furthermore, as integrated genomes evolve neutrally, or almost neutrally ${ }^{29}$, they cannot produce a phylogenetic signal incompatible with that of the host genome. Because of its peculiar integrating genome biology, we nonetheless advocate caution in the interpretation of our results for wAna.

Rates of Wolbachia evolution correlates with nuclear, but not with mitochondrial genome of hosts. An intriguing outcome of our co-phylogenies is the striking heterogeneity of the evolutionary rate of Wolbachia compared to that of the host's mitochondrial DNA (mtDNA). While we could reproduce previous finding that Wolbachia evolves about one order of magnitude (circa 10 times) slower than mitochondria in D. melanogaster ${ }^{16}$, we could observe a similar pattern only in D. mauritiana, and to a lesser extent in $D$. simulans and $D$. ananassae. In all other hosts we observe different patterns: Wolbachia rates are approximately the same as mitochondria in Onchocerca nematodes, and two/ three times faster than mitochondria in the wasp $D$. alloeum, the beetle $D$. virgifera and the bedbug $C$. lecturalis. These discrepancies seem to correlate with host biology traits such as generation time and population size: while Drosophila, for example, is characterized by many generations per year and large effective population size ${ }^{35}$, most other species are characterized by less generation per year and smaller population size $e^{36,37}$.

Our co-phylogenies reveal major desynchronization between the Wolbachia and the mtDNA clocks. We did not infer cophylogenies using host nuclear data because, while mtDNA and Wolbachia are uniparentally inherited, nuclear genes follow a Mendelian inheritance and their coalescence impedes building a genealogical (tree-like) structure ${ }^{38}$. We have however estimated average genetic divergence for a typical universal animal nuclear marker, the $18 \mathrm{~S}$ rRNA gene ${ }^{5}$ and compared the average genetic distances of each of the 11 Wolbachia populations with that of the mtDNA and 18S of the corresponding hosts. As anticipated by the different relative rates of the co-phylogenies (Fig. 4a), there is no correlation between Wolbachia and mtDNA divergences (Fig. 4b). Instead, we found a significant correlation between Wolbachia and 18S rRNA gene evolution (Fig. 4b). This indicates that Wolbachia is indeed following the molecular clock of the hosts, but only for what concerns its nuclear genome, not the mitochondrial one. By contrast, the mtDNA seems to be characterized by a rate that departs from both Wolbachia and, apart from a few cases, the nuclear data. One possible explanation is that the mitochondrial genomes of 4-5 species are characterized by an unusual within-species genetic homogeneity possibly due to specific inheritance genetics or a constrained selective regime. Indeed, when we exclude these species (circled in Fig. 4b), we recover a significant correlation, and notably with a similar slope of the Wolbachia-18S correlation (dotted line in Fig. 4b). This finding deserves future testing in particular by analyzing large chunks of the host nuclear genome and by expanding these comparisons to more populations. Our data is however clear in showing that the pattern and rate of Wolbachia evolution may be very dissimilar to that of their mtDNA host, pointing at a variably and species-specific Wolbachia-mtDNA evolution, in which the 
Drosophila-Wolbachia dynamics ${ }^{16}$ seems to be the exception rather than the norm.

\section{Discussion}

We have investigated the global Wolbachia genomic diversity by de novo assembly and analysis of more than a thousand genomes. With 1.793 positive samples and 1005 assembled genomes, 40 of which are from new host species, we show that our metagenomics approach is effective in retrieving large amounts of endosymbiont genome data. Overall, we could show that our approach provides a highly robust enhanced picture of both deep time and shallow time Wolbachia evolution, which may be used as an actual reference for future studies. We advocate that the metagenomic approach and pipeline we have used here should be extended to other less known unculturable endosymbionts such as Spiroplasma or Cardinium ${ }^{40}$.

Our data allowed us to explore more in depth Wolbachia diversity. We found new variants related to model Wolbachia strains such as $w \mathrm{Mel}$ and $w \mathrm{Ri}$ as well as new genomes from medically and socio-economically relevant organisms including mosquitoes, pests of agriculture, and nematodes. Our phylogenies provide a robust reference for future Wolbachia studies and the branching position of parasites and parasitoids in our phylogenies invigorate the idea that they may have played a key role in the horizontal transfer of this bacteria.

Probably the most exciting outcome of our study is the between-hosts heterogeneity of Wolbachia evolution rate in comparison to mitochondrial rate: in some hosts Wolbachia evolves 10 times slower than mitochondria, in other hosts at the same rate or even faster than mitochondria. We hypothesize that the reason behind this large rate variability is not due to Wolbachia, but rather due to peculiar mitochondrial genetics in some hosts: this is because rates of Wolbachia evolution correlates with nuclear, but not with mitochondrial genome of hosts. This has important outcomes for comparative studies: mutation rate inferred from the mitochondrial rate of model organisms such as D. melanogaster ${ }^{16}$ should be used with care when calibrating Wolbachia evolution in other hosts ${ }^{39}$.

\begin{abstract}
Methods
Retrieval of existing Wolbachia reference genomes. The reference set of Wolbachia genomes was compiled with the sequenced strains publicly available as of January 2017. We retrieved 41 of the 43 public available Wolbachia reference genomes from RefSeq ${ }^{41}$ and GenBank ${ }^{42}$, whereas the genomes of strains wDi and wLs which were downloaded from http://nematodes.org/genomes/index_filaria.html 43 because these two genomes were not yet deposited in NCBI. This set includes 19 genomes of Wolbachia supergroup A, 15 of supergroup B, four of supergroup C, three of supergroup D, one of supergroup F, and one of supergroup L. The NCBI accession numbers for all Wolbachia genome sequences used for phylogenetic analysis are given in Supplementary Data 3. To avoid potential biases due to different genome annotation procedures, all genomes were re-annotated using PROKKA ${ }^{44}$ (version 1.11).
\end{abstract}

Retrieval of raw-sequencing data from host (re)sequencing projects. Public shotgun sequencing samples were retrieved from the NCBI Sequence Read Archive (SRA) database by selecting all available sequencing projects that targeted organisms in taxonomic families that can host Wolbachia endosymbionts. The database search was based on 181 arthropod and nematode host-related keywords (common names such as insect(s), worm(s), mite(s), plus various taxonomic units ranging from Phyla to Genera; see Supplementary Information) and resulted in a total number of 31,825 samples (72.3TB of raw-sequencing data, Supplementary Data 4)).

Identification of Wolbachia-positive samples. We first screened for the presence of Wolbachia in the samples to identify the samples that were analyzed with the genome and pangenome reconstruction pipelines (see below). In this screening step, the sequences of all samples were mapped against available Wolbachia reference genomes using the Bowtie $2^{45}$ aligner (version 2.1.0). Breadth of coverage for each reference genome was calculated using Samtools ${ }^{46}$. For follow-up analysis, we selected the 1,793 samples that show a breadth of coverage larger than $50 \%$ of at least one reference genome (at $1 \mathrm{X}$ depth of coverage level). All these samples were used for the pangenome analysis $\left(\mathrm{PanPhlAn}^{24}\right)$. The 1220 samples with an average depth of coverage for a Wolbachia genome larger than $4 \mathrm{X}$ were selected for the assembly-based approach.

Wolbachia prevalence and estimated titers. For host species with multiple screened samples, we identified the Wolbachia prevalences as the proportion of infected subjects found in a host species population (Fig. 1). Prevalences are estimated for strain detection at a low $>1 \mathrm{X}$ coverage level using PanPhlAn and for higher $>10 \mathrm{X}$ coverage as required for assembly. Supplementary Data 4) includes the prevalences for all host species in which we could detect at least one Wolbachia strain. Non-infected (zero prevalence) host species are only included for large screens of 50 or more samples from the same host. The reported prevalence levels might be affected by the sometimes limited metadata quality of the NCBI repository and our sampling strategy. Host species tree topology and divergences (Fig. 1) are obtained following Rota Stabelli et al. ${ }^{5}$ and Ometto et al. ${ }^{35}$. Infectious titers are estimated for each assembled Wolbachia genome based on the abundance ratio between Wolbachia and host sequences, and normalized by host and Wolbachia genome lengths (Supplementary Data 3).

Assembly-free Wolbachia pangenome analysis using PanPhIAn. We applied PanPhlAn ${ }^{24}$ to characterize the strain-specific gene repertoire of the Wolbachia genomes in the positive samples. In its first step, PanPhlAn estimates the abundances of all genes in the Wolbachia pangenome. Then, it uses a co-abundance criterion to define the set of genes specific to the strain present in a sample. The Wolbachia pangenome, consisting of all genes known to occur in a Wolbachia strain, was generated by merging genes from all 43 Wolbachia reference genomes. Homologous genes are clustered into gene families with a nucleotide identity threshold of $80 \%$ using Roary ${ }^{47}$, version 3.5 .9 , which resulted into a set of 10,725 distinct pangenes. For all 1793 pre-selected samples, pangene profiles were generated using a minimum coverage threshold of 1X ("-min_coverage 1" option) which allow profiling also those strains that cannot be assembled because of low coverage.

\section{Wolbachia (metagenomic) assembly and annotation from host sequencing}

data. We applied metagenomic assembly to each of the 1220 Wolbachia-positive samples having a coverage of at least 4X. 1005 MAGs passed our quality control criteria as defined below. Since some MAGs of lower quality are derived from exceptional hosts, for reconstructing the phylogenetic core tree, we used all 1161 MAGs that contain at least 33\% core genes (Supplementary Data 5). 54 MAGs are rejected as not fulfilling any quality criteria. Assembly was performed with $\mathrm{MegaHit}^{48}$ version 1.0.5. Contigs shorter than $1000 \mathrm{nt}$ were removed. Because of the availability of a large set of Wolbachia reference genomes, we used a taxonomybased binning approach to group contigs into draft genomes. This pipeline starts by mapping all the assembled contigs against all Wolbachia reference genomes using BLASTn ${ }^{49}$ with the "task blastn" option that uses a shorter word-size of 11 $\mathrm{bp}$, thereby allowing to align more distantly related sequences. A contig was included into a Wolbachia draft genome if it mapped against any Wolbachia reference genome with $>75 \%$ identity over a fraction of the length of the contig higher than a threshold $\mathrm{T}$. The value of $\mathrm{T}$ was chosen so that short contigs were required to overlap almost entirely with the reference while longer contigs were only required to align at a shorter fraction with a reference genome in order to allow the discovery of new Wolbachia accessory sequences. We thus used a logarithmically scaled value such than short $1000 \mathrm{bp}$ contigs were required to be aligned over the full length of the contig, 2,000 bp contigs over $65 \%$ of the length, and contigs longer than 10,000 bp over $20 \%$ of the length. Precisely, for a contig with a total length $L_{\mathrm{c}}$ and aligned length $L_{\mathrm{a}}$, we require: $L_{\mathrm{a}} / \log _{10}\left(L_{\mathrm{c}} / 100\right) / 1000>1$ The final contig sets of assembled Wolbachia draft genomes were annotated using PROKKA. Information about the draft genomes, including the NCBI source sample SRA number and host species, are given in Supplementary Data 5.

Quality control of the assembled Wolbachia genomes. To select quality controlled genomes, we filtered out assembled Wolbachia genomes that (i) showed a genome coverage lower than $20 \mathrm{X}$, (ii) contained less than $50 \%$ core genes (see below), (iii) had a total length shorter or longer than the range 750,000-1,700,000 bp (set based on the lengths of Wolbachia reference genomes), and finally (iv) showed evidence of chimeric assemblies due to the presence in the corresponding sample of more than one Wolbachia strain. For detecting Wolbachia coinfections, point (iv), we generalized the approach we described for StrainPhlAn ${ }^{50}$ to identify polymorphisms in the reads used to build the assemblies. The number of polymorphic sites in the assembled genomes gives a direct indication of the presence of one or more Wolbachia genomes in the same sample. A high polymorphic rate can indicate potential incorporation of multiple populations into a single MAG or incorporation of host sequence of lateral gene transfer (LGT) events. In detail, the procedure starts by mapping with Bowtie $2^{45}$ all the reads of a sample against the Wolbachia genome assembled from the same sample. The resulting alignment was processed using Samtools ${ }^{46}$ to detect polymorphisms in the reads mapping against each position of the assembly. Based on a probability mass function of a binomial distribution, we label as polymorphic those positions for which we reject the null hypothesis (alpha 0.05 ) that only one base is present. We consider an error rate of 
0.01 (i.e. 1\%) for Illumina sequencing reads and we only consider read bases with a quality higher than 30 . Once all positions of the assembly are assessed, we consider the fraction of the assembly length with significant polymorphisms. When this fraction is greater than 0.05 (more than $5 \%$ polymorphic sites) we assume that multiple Wolbachia is present in the same sample. Assembled genomes are thus considered as of reasonable quality when the polymorphic rate is lower than 0.01 or between 0.01 and 0.05 but showing a dominance of the primary strain in the polymorphic regions larger than $80 \%$. Polymorphic rates are shown in Supplementary Fig. 2. Additionally, we screened our assemblies for low-confidence regions defined by unexpected higher coverage and higher sequence variation compared to average, following the procedure of Chung et al. ${ }^{34}$. We extracted all $\geq 50$ bp regions with a sequencing depth of $\geq 4$ median coverage and all $\geq 50 \mathrm{bp}$ regions with $\geq 4$ average sequence variation (positions with secondary base $\geq 5 \%$ coverage). To control for potential sequence contamination, we mapped all our passed QC assemblies against nearly 100,000 bacteria reference genomes from NCBI GenBank. Using a BLAST threshold of $>97 \%$ identity over a minimum alignment length of 10,000 nucleotides, we identified only one Drosophila melanogaster related Wolbachia strain (SRR1183652) that includes sequences that are similar to Acetobacter related species. Additionally, we could confirm the quality of our assemblies by re-assembling Wolbachia reference genomes from available source samples. Based on a core sequence length of more than $300,000 \mathrm{nt}$, we found 99.99\% genetic identity between our assemblies (SRR183690, SRR1508956) and the original reference genomes of $w \mathrm{Di}$ and $w \mathrm{Rec}$, and even $100 \%$ core identity between our assembly (ERR188908) and the reference strain $w$ Suzi. To confirm NCBI metadata about the host and to clarify samples tagged as "Apoidea" and "Insecta", we verified the host species by reconstructing the $18 \mathrm{~S}$ ribosomal RNA gene sequence using the tool RiboTagger ${ }^{51}$. Based on all these criteria, we selected 1005 assemblies that fulfilled our quality requirements. The assembly quality measures for the genomes are given in Supplementary Data 5

Pangenome analysis and core-genome identification. In a first step, we generated the Wolbachia pangenome across all assemblies using Roary ${ }^{47}$ version 3.5.9 with a BLAST identity cutoff of $80 \%$ and paralog splitting disabled. We then identified the Wolbachia core genes by applying an approach that balances the different numbers of assembled genomes in the Wolbachia supergroups. To get an unbiased set of core genes, we selected a set of five representative genomes from each supergroup as follows: We clustered all genomes of each supergroup using $k$ medoids clustering ( $k=5$, Jaccard distance) applied on Roary gene presence/ absence profiles. The central genomes of each of the five clusters are selected as representative genomes of a supergroup, which in total are 25 genomes covering the variation of Wolbachia supergroups A, B, C, D, and F. We generated the pangenome of the 25 representative genomes using Roary with a BLAST identity cutoff of $80 \%$ and paralog splitting disabled. The final core gene set was created by selecting gene-families that are present in at least one of the five representative genomes in each Wolbachia supergroups. In total, we could identify 316 Wolbachia core genes from the representative genomes present in all considered supergroups. We then used our Wolbachia core gene set to compare all 1200 assembled genomes by extracting the individual core gene sequence for each genome. For each core gene-family, we mapped the gene sequences of the 25 representative genomes against the gene sequences of each genome using BLASTn (task type blastn and word-size $=7 \mathrm{bp}$ ). A matched sequence was selected as core gene sequence based on the following thresholds: E-value $<1 \mathrm{e}-10$, percent identity $>66 \%$, and the alignment length was required to be not shorter than $50 \%$ of the average core gene sequence and not longer than $125 \%$. To identify single-copy genes, we excluded three core genes that show multiple hits in more than ten percent of our assembled genomes. For all remaining 313 core genes, we selected only gene sequences that are present as a single hit.

Phylogenetic analysis. Based on the identified Wolbachia core gene set, we constructed a comprehensive phylogenetic tree covering five different Wolbachia supergroups by using all assembled genomes that contain at least 33\% core genes. We aligned each core gene family using $\mathrm{Mafft}^{52}$ version 7.215 with globalpair G-INS-i strategy and we then concatenated the alignments. We constructed the maximum-likelihood tree (Fig. 2a) under the GTR plus GAMMA model using RAxML $^{53}$ version 8.2.9. Node support was estimated with 100 bootstrap replicates.

Functional analysis. To compare the gene content of our assembled Wolbachia strains, we characterized the core and accessory genome of the overall Wolbachia population and compared 14 host-specific lineages. Comparison is based on Roary gene clustering as described above. Fisher exact test $(p<0.05)$ was used to identify lineage-specific gene gain and loss (Fig. 3, Supplementary Data 6). Each host-specific lineage was separately compared to all closely related lineages. Gain or loss of genes are only reported if coincident across all comparisons. To identify CI genes, we compared non-CI $w$ Au-like genomes independently with both CI lineages $w$ Ri-like and $w$ Mel, excluding 79 genomes that show an identical core sequence to other genomes. Only genes that are significantly enriched in both comparisons are reported as CI candidate genes. Additional comparisons of both CI lineages with nematode lineages (B. malayi, O. ochengi) are performed to reject incorrect $\mathrm{CI}$ gene loss due to genes enriched in the nonCI $w$ Au-like lineage.

Co-phylogenies including MUMmer alignments and mitogenomes. Hostspecific multiple genome alignments of Wolbachia sequences were constructed for the 11 species in Fig. 4 using a modified StrainEst ${ }^{54}$ pipeline. Briefly, for each host species a reference genome of Wolbachia was chosen and all other genomes were aligned against the reference using the nucmer command from the MUMmer suite $^{55}$. Ambiguous mappings (i.e., regions that can be mapped against more than one region) in alignments were discarded. In order to reduce the size of the datasets in the case of strains infecting D. melanogaster and of wRi strains infecting $D$. simulans, genomes were filtered by discarding those of total length $<1.1 \mathrm{Mbp}$. In addition, since aligning a large number of almost identical draft genomes could severely reduce the size of the core genome due to the stochastic distribution of missing sequences, for these two datasets an all-vs-all distance matrix was computed using $\mathrm{Mash}^{56}$, and sequences were clustered using complete linkage hierarchical clustering with a threshold of 0.005 , keeping only one representative sequence for each cluster for downstream analysis. From the nucmer pairwise sequence alignments to the reference a host-specific core genome alignment was built.

We reconstructed the mitochondrial DNA (mtDNA) of eleven hosts by using a reference-based read mapping. Short reads were aligned with the corresponding host mtDNA reference using Bowtie2. A consensus sequence was built from all covered reads using samtools mpileup and a majority rule to merge the bases across reads. Host-specific mtDNA sequences were subsequently aligned using Mafft with globalpair G-INS-i strategy.

We inferred phylogenies of the eleven sets of Wolbachia and corresponding mitochondrial genomes using BEAST ${ }^{57}$ version 1.10 and employing for both types of datasets a GTR $+\mathrm{G}$ replacement model, a coalescent tree prior, strict clock, and no calibrations priors in order to have phylogenies scaled only on mutations per site. We repeated the analyses using RAxML employing a GTR plus GAMMA replacement model (as detailed above) to further test phylogenies. For computational reasons, we inferred BEAST analyses of Wolbachia from $D$. melanogaster and D. simulans after exclusion of invariable sites: for consistencies, tree topologies have been rescaled according to RAxML branch lengths inferred using whole alignment.

Reporting summary. Further information on research design is available in the Nature Research Reporting Summary linked to this article.

\section{Data availability}

All assembled Wolbachia genomes described in Fig. 2 have been deposited at the European Nucleotide Archive (ENA) under project PRJEB35167. All trees and corresponding xml files are in Supplementary Data 1-2. Reference genomes, download statistics, individually assembled genome with accession numbers and statistics, functional data, and genetic distances are provided in Supplementary Data 3-7).

\section{Code availability}

All relevant codes used in this work are available, upon request, from the corresponding authors (N.S. or O. R-S).

Received: 18 April 2019; Accepted: 25 September 2020; Published online: 16 October 2020

\section{References}

1. Florez, L. V. et al. Antibiotic-producing symbionts dynamically transition between plant pathogenicity and insect-defensive mutualism. Nat. Commun. 8, 15172 (2017)

2. Nyholm, S. V. \& McFall-Ngai, M. J. The winnowing: establishing the squidvibrio symbiosis. Nat. Rev. Microbiol. 2, 632-642 (2004).

3. Van Deynze, A. et al. Nitrogen fixation in a landrace of maize is supported by a mucilage-associated diazotrophic microbiota. PLoS Biol. 16, e2006352 (2018).

4. Werren, J. H., Baldo, L. \& Clark, M. E. Wolbachia: master manipulators of invertebrate biology. Nat. Rev. Microbiol. 6, 741-751 (2008).

5. Rota-Stabelli, O., Daley, A. C. \& Pisani, D. Molecular timetrees reveal a Cambrian colonization of land and a new scenario for ecdysozoan evolution. Curr. Biol. CB 23, 392-398 (2013).

6. Gerth, M. \& Bleidorn, C. Comparative genomics provides a timeframe for Wolbachia evolution and exposes a recent biotin synthesis operon transfer. Nat. Microbiol. 2, 16241 (2016).

7. Weinert, L. A., Araujo-Jnr, E. V., Ahmed, M. Z. \& Welch, J. J. The incidence of bacterial endosymbionts in terrestrial arthropods. Proc. Biol. Sci. 282, 20150249 (2015).

8. McGraw, E. A. \& O’Neill, S. L. Beyond insecticides: new thinking on an ancient problem. Nat. Rev. Microbiol. 11, 181-193 (2013). 
9. Hughes, G. L. \& Rasgon, J. L. Transinfection: a method to investigate Wolbachia-host interactions and control arthropod-borne disease. Insect Mol. Biol. 23, 141-151 (2014).

10. Nikolouli, K. et al. Sterile insect technique and Wolbachia symbiosis as potential tools for the control of the invasive species Drosophila suzukii. J. Pest Sci. 91, 489-503 (2018).

11. LePage, D. P. et al. Prophage WO genes recapitulate and enhance Wolbachiainduced cytoplasmic incompatibility. Nature 543, 243-247 (2017).

12. Beckmann, J. F., Ronau, J. A. \& Hochstrasser, M. A Wolbachia deubiquitylating enzyme induces cytoplasmic incompatibility. Nat. Microbiol. 2, 17007 (2017).

13. Baldo, L. et al. Insight into the routes of Wolbachia invasion: high levels of horizontal transfer in the spider genus Agelenopsis revealed by Wolbachia strain and mitochondrial DNA diversity. Mol. Ecol. 17, 557-569 (2008).

14. Bailly-Bechet, M. et al. How long does Wolbachia Remain on board? Mol. Biol. Evol. 34, 1183-1193 (2017).

15. Schuler, H. et al. The hitchhiker's guide to Europe: the infection dynamics of an ongoing Wolbachia invasion and mitochondrial selective sweep in Rhagoletis cerasi. Mol. Ecol. 25, 1595-1609 (2016).

16. Richardson, M. F. et al. Population genomics of the Wolbachia endosymbiont in Drosophila melanogaster. PLoS Genet. 8, e1003129 (2012).

17. Choi, Y. J. et al. Genomic diversity in Onchocerca volvulus and its Wolbachia endosymbiont. Nat. Microbiol. 2, 16207 (2016).

18. Signor, S. Population genomics of Wolbachia and mtDNA in Drosophila simulans from California. Sci. Rep. 7, 13369 (2017).

19. Kaur, R., Siozios, S., Miller, W. J. \& Rota-Stabelli, O. Insertion sequence polymorphism and genomic rearrangements uncover hidden Wolbachia diversity in Drosophila suzukii and D. subpulchrella. Sci. Rep. 7, 14815 (2017).

20. Bleidorn, C. \& Gerth, M. A critical re-evaluation of multilocus sequence typing (MLST) efforts in Wolbachia. FEMS Microbiol. Ecol. 94 https://doi.org/ 10.1093/femsec/fix163 (2018).

21. Kumar, N. et al. Efficient enrichment of bacterial mRNA from host-bacteria total RNA samples. Sci. Rep. 6, 34850 (2016).

22. Salzberg, S. L. et al. Serendipitous discovery of Wolbachia genomes in multiple Drosophila species. Genome Biol. 6, R23 (2005).

23. Pascar, J. \& Chandler, C. H. A bioinformatics approach to identifying Wolbachia infections in arthropods. PeerJ 6, e5486 (2018).

24. Scholz, M. et al. Strain-level microbial epidemiology and population genomics from shotgun metagenomics. Nat. Methods 13, 435-438 (2016).

25. Chrostek, E. \& Gerth, M. Is Anopheles gambiae a Natural Host of Wolbachia? mBio 10, e00784 (2019).

26. Gerth, M., Saeed, A., White, J. A. \& Bleidorn, C. Extensive screen for bacterial endosymbionts reveals taxon-specific distribution patterns among bees (Hymenoptera, Anthophila). FEMS Microbiol. Ecol. 91 https://doi.org/ 10.1093/femsec/fiv047 (2015).

27. Segata, N., Bornigen, D., Morgan, X. C. \& Huttenhower, C. PhyloPhlAn is a new method for improved phylogenetic and taxonomic placement of microbes. Nat. Commun. 4, 2304 (2013).

28. Pisani, D. et al. Genomic data do not support comb jellies as the sister group to all other animals. Proc. Natl Acad. Sci. USA 112, 15402-15407 (2015).

29. Choi, J. Y., Bubnell, J. E. \& Aquadro, C. F. Population Genomics of Infectious and Integrated Wolbachia pipientis Genomes in Drosophila ananassae. Genome Biol. Evol. 7, 2362-2382 (2015).

30. Meany, M. K. et al. Loss of cytoplasmic incompatibility and minimal fecundity effects explain relatively low Wolbachia frequencies in Drosophila mauritiana. Evolution 73, 1278-1295 (2019).

31. Cooper, B. S., Vanderpool, D., Conner, W. R., Matute, D. R. \& Turelli, M. Introgressive and horizontal acquisition of Wolbachia by Drosophila yakubaclade hosts and horizontal transfer of incompatibility loci between distantly related Wolbachia. bioRxiv https://doi.org/10.1101/551036 (2019).

32. Turelli, M. et al. Rapid global spread of wRi-like Wolbachia across multiple Drosophila. Curr. Biol. CB 28, 963-971 e968 (2018).

33. Kriesner, P., Hoffmann, A. A., Lee, S. F., Turelli, M. \& Weeks, A. R. Rapid sequential spread of two Wolbachia variants in Drosophila simulans. PLoS Pathog. 9, e1003607 (2013).

34. Chung, M., Small, S. T., Serre, D., Zimmerman, P. A. \& Dunning Hotopp, J. C. Draft genome sequence of the Wolbachia endosymbiont of Wuchereria bancrofti wWb. Pathogens Dis. 75, ftx115 (2017).

35. Ometto, L. et al. Linking genomics and ecology to investigate the complex evolution of an invasive Drosophila pest. Genome Biol. Evol. 5, 745-757 (2013).

36. Etya'ale, D. Vision 2020: update on onchocerciasis. Community Eye Health 14, 19-21 (2001)

37. Aak, A. \& Rukke, B. A. Bed bugs, their blood sources and life history parameters: a comparison of artificial and natural feeding. Med. Vet. Entomol. 28, 50-59 (2014).

38. Benazzo, A. et al. Survival and divergence in a small group: the extraordinary genomic history of the endangered Apennine brown bear stragglers. Proc. Natl Acad. Sci. USA 114, E9589-E9597 (2017).
39. Conner, W. R. et al. Genome comparisons indicate recent transfer of wRi-like Wolbachia between sister species Drosophila suzukii and D. subpulchrella. Ecol. Evol. 7, 9391-9404 (2017)

40. Brown, A. M. V. et al. Comparative genomics of Wolbachia-Cardinium dual endosymbiosis in a plant-parasitic nematode. Front. Microbiol. 9, 2482 (2018).

41. O'Leary, N. A. et al. Reference sequence (RefSeq) database at NCBI: current status, taxonomic expansion, and functional annotation. Nucleic Acids Res. 44, D733-D745 (2016).

42. Benson, D. A. et al. GenBank. Nucleic Acids Res. 45, D37-D42 (2017).

43. Comandatore, F. et al. Phylogenomics and analysis of shared genes suggest a single transition to mutualism in Wolbachia of nematodes. Genome Biol. Evol. 5, 1668-1674 (2013).

44. Seemann, T. Prokka: rapid prokaryotic genome annotation. Bioinformatics 30, 2068-2069 (2014).

45. Langmead, B. \& Salzberg, S. L. Fast gapped-read alignment with Bowtie 2. Nat. Methods 9, 357-359 (2012).

46. Li, H. et al. The sequence alignment/map format and SAMtools. Bioinformatics 25, 2078-2079 (2009).

47. Page, A. J. et al. Roary: rapid large-scale prokaryote pan genome analysis. Bioinformatics 31, 3691-3693 (2015).

48. Li, D. et al. MEGAHIT v1.0: A fast and scalable metagenome assembler driven by advanced methodologies and community practices. Methods 102, 3-11 (2016).

49. Altschul, S. F., Gish, W., Miller, W., Myers, E. W. \& Lipman, D. J. Basic local alignment search tool. J. Mol. Biol. 215, 403-410 (1990).

50. Truong, D. T., Tett, A., Pasolli, E., Huttenhower, C. \& Segata, N. Microbial strain-level population structure and genetic diversity from metagenomes. Genome Res. 27, 626-638 (2017).

51. Xie, C., Goi, C. L., Huson, D. H., Little, P. F. \& Williams, R. B. RiboTagger: fast and unbiased $16 \mathrm{~S} / 18 \mathrm{~S}$ profiling using whole community shotgun metagenomic or metatranscriptome surveys. BMC Bioinform. 17, 508 (2016).

52. Katoh, K. \& Standley, D. M. MAFFT multiple sequence alignment software version 7: improvements in performance and usability. Mol. Biol. Evol. 30, 772-780 (2013)

53. Stamatakis, A. RAxML version 8: a tool for phylogenetic analysis and postanalysis of large phylogenies. Bioinformatics 30, 1312-1313 (2014).

54. Albanese, D. \& Donati, C. Strain profiling and epidemiology of bacterial species from metagenomic sequencing. Nat. Commun. 8, 2260 (2017).

55. Kurtz, S. et al. Versatile and open software for comparing large genomes. Genome Biol. 5, R12 (2004).

56. Ondov, B. D. et al. Mash: fast genome and metagenome distance estimation using MinHash. Genome Biol. 17, 132 (2016).

57. Suchard, M. A. et al. Bayesian phylogenetic and phylodynamic data integration using BEAST 1.10. Virus Evol. 4, vey016 (2018).

\section{Acknowledgements}

The authors would like to thank Hannes Schuler for helpful comments on the manuscript. This work was supported by ERC (MetaPG-716575), MIUR (RBFR13EWWI), EU-FP7 (PCIG13-GA-2013-618833), CARITRO (2013.0239), and LEO Pharma Foundation to N.S.

\section{Author contributions}

O.R.S., N.S., and M.S. designed research, M.S. performed retrieval, filtering, and assembly of genomes, M.S. and K.T. performed functional analyses, M.S., D.A., and C.D. performed whole-genome alignments, M.S. and O.R.S. performed phylogenetic analyses. O.R.S., M.S., and N.S. drafted the manuscript. All authors edited and commented on the manuscript.

\section{Competing interests}

The authors declare no competing interests.

\section{Additional information}

Supplementary information is available for this paper at https://doi.org/10.1038/s41467 020-19016-0.

Correspondence and requests for materials should be addressed to N.S. or O.R.-S.

Peer review information Nature Communications thanks Michael Gerth, Benjamin Tully and the other, anonymous, reviewer for their contribution to the peer review of this work. Peer reviewer reports are available.

Reprints and permission information is available at http://www.nature.com/reprints

Publisher's note Springer Nature remains neutral with regard to jurisdictional claims in published maps and institutional affiliations. 
(c) (i) Open Access This article is licensed under a Creative Commons Attribution 4.0 International License, which permits use, sharing, adaptation, distribution and reproduction in any medium or format, as long as you give appropriate credit to the original author(s) and the source, provide a link to the Creative Commons license, and indicate if changes were made. The images or other third party material in this article are included in the article's Creative Commons license, unless indicated otherwise in a credit line to the material. If material is not included in the article's Creative Commons license and your intended use is not permitted by statutory regulation or exceeds the permitted use, you will need to obtain permission directly from the copyright holder. To view a copy of this license, visit http://creativecommons.org/ licenses/by/4.0/.

(C) The Author(s) 2020 